\title{
Peter Careys Kurzgeschichten: Gefangen in einem erzählerischen Labyrinth
}

\section{Cornelia Schulze, Bergische Universität Wuppertal}

Throughout Carey's fiction, both literal and figurative entrapment are invoked; [...]. His fictions abound with actual prisons and cages, which perhaps serve as metonym for Australian society. But he is also fascinated by the construction of entrapping structures, [...], and often that involves individual, figuratively entrapping structures. (Fletcher 74)

Nicht nur Peter Careys Romane, sondern auch seine Kurzgeschichten beinhalten vielfach einschränkende Situationen, Orte oder Ereignisse. Denn in seinen Erzählungen entwirft Carey fiktive Welten, die ihrer eigenen Logik folgen und surreale, fabelhafte und bizarre Elemente enthalten. Meist handelt es sich dabei um Distopien, in denen die Protagonisten gefangen sind. Gleichzeitig manipuliert Carey sein Verhältnis zum Leser derart, dass sich dieser ähnlich gefangen oder ausgeliefert fühlt, wie die Protagonisten der fiktiven Welt. Dieser Essay soll aufzeigen, welche Formen von Gefangenschaft Carey in seiner Kurzprosa präsentiert und wie es ihm gelingt, den Leser in seine klaustrophobischen Visionen hinein zu ziehen. Eine Neubewertung seiner Intention legt jedoch nahe, dass und auf welche Weise diese 'doppelte Gefangennahme' letztendlich zur Befreiung führen kann.

\section{Peter Careys erzählerische Kunstgriffe: Ein verändertes Autor-Leser Verhältnis}

Peter Careys erzählerisches Talent ist unbestritten (vgl. Kane 519, Todd 305, Hassall 637ff), und sein unverwechselbarer Erzählstil wurde in der Zeit geprägt, als er als Texter in einer Werbeagentur arbeitete:

Like a number of his contemporaries, including Murray Bail, Morris Lurie and Barry Oakley, Carey came from the world of advertising copywriting, a world in which the writer as prophet, the writer as visionary, the writer as moralist, the writer as historian, had been superseded by the writer as manipulator, the writer as technician of special effects. The skill lay in producing a powerful reader response. This was the post-modern aesthetic of the game, the enigma, the construct, the product. (Wilding xiv-xv).

Dieses spielerisch-manipulative Verhältnis, das zwischen Werbetextern und potentiellen Käufern üblich ist, kennzeichnet auch das Verhältnis zwischen dem Schriftsteller Carey und seiner Leserschaft. Für Ryan-Fazilleau führt diese Erzählweise jedoch zu einem frustrierenden Leseerlebnis, welches das traditionelle Vertrauensverhältnis zwischen Autor und Leser gefährdet: "In Carey's short stories the reader finds himself on unfamiliar terrain, for $[. .$.$] the author deliberately and constantly undermines our confidence in him, his text$ and ourselves. This approach subverts the symbiotic type of author/reader relationship we are used to" (51). Als Beweise für die veränderte Autor-Leser-Beziehung führt die Autorin vier narrative Verunsicherungsstrategien an, die Carey innerhalb seiner Kurzgeschichten nutzt: (1) Careys Protagonisten machen Äußerungen, die nicht zu decodieren seien; aber dennoch strafe er den Leser für seine Unfähigkeit mit Häme. (2) Carey schüre Unverständnis, indem er den Leser mithilfe wiederholter Referenzen/Motive auf eine falsche Fährte lockt. (3) Carey betone explizit die Unglaubwürdigkeit des Erzählers. (4) Carey dringe in die Domäne des aufgeklärten Lesers ein, indem er ihm vorgebe, welche Fragen er sich zu stellen habe 
und zu welchen interpretatorischen Ergebnissen er kommen müsse (vgl. 51-58). Erst mit der Analyse der Verunsicherungsstrategien sei das Kräfteverhältnis, das zu Ungunsten des Lesers manipuliert wurde, wieder hergestellt. Die Autorin vermutet, dass Carey sich Leser wünscht, die sich als würdige Gegner erweisen: "He wants an active opponent of a playing standard (almost) equal to his own" (62). Auch wenn sie diesen Essay mit seinen Spiel- und Kriegsmetaphern mit einem Augenzwinkern und Bewunderung für den "brilliant and diabotical Carey" (57) verfasst hat, unterstellt sie Carey Manipulation als Selbstzweck bzw. zur Belustigung des Autors. Es ist jedoch auch eine andere Wertung von Careys Erzählverhalten möglich. Dieser Essay geht von der These aus, dass Carey seine Leser zwar bewusst manipuliert, sie aber auf diese Weise dafür sensibilisieren möchte, wie in der heutigen westlichen Welt die Menschen zu Opfern eines repressiven Systems werden: dem Spätkapitalismus. Um die perfiden Mechanismen der Unterdrückung zu entlarven, überträgt Carey diese inhaltlich (als Motiv des Gefangenseins) wie formal (durch manipulative Lesersteuerung) auf seine Erzählungen. Careys Erzählungen fordern den Leser somit heraus, nicht nur die Welt der Fiktion, sondern auch die reale Welt zu durchschauen.

\section{Peter Careys Kurzgeschichten als Lehrstücke}

Careys Kurzgeschichten können demnach als Lehrstücke zur heutigen Zeit gewertet werden. In einem Interview äußert sich Carey besorgt, da aus seiner Sicht viele Menschen unter albtraumhaften Umständen leben, diese jedoch selten erkennen, geschweige denn zu ändern suchen: "People often live in nightmares without knowing it. The nightmare creeps up on them and even when it's at its most intense it feels quite normal to them. Not nice, but normal" (Carey in Ikin 33). In Peter Careys Wirklichkeitsentwürfen verbinden sich Surrealismus und Realismus zu einer ebenso albtraumhaften Welt, in der sich Careys Charaktere entweder mit schlafwandlerischer Sicherheit in Sackgassen manövriert haben oder sich in Situationen wieder finden, die sie selbst nicht zu verantworten, wohl aber zu ertragen haben. Während einige der Charaktere aussichtslos gegen ihre Lebensbedingungen rebellieren, nehmen die meisten ihre Situation in fatalistischer Haltung an. Als Ursache für die Akzeptanz des alltäglichen Wahns nennt Carey Entfremdung, die Produkt des spätkapitalistischen Systems sei:

We are alienated from each other, from ourselves, work, from our environment. We are denied access to information and given misinformation instead. We are raised within an authoritarian system and teach our children to look for leaders. [...]. I don't think people are mindless or stupid and no matter how fucked around we are by the values of late capitalism, I still think there is some residual human decency in most of us. (Carey in Neilsen 72)

Ähnlich wie in unserer medial gesteuerten Alltagswelt Informationen zurück gehalten oder verfälscht werden, so bedient sich Carey Erzähltechniken, die den Leser zunächst irreführen. Während Ryan-Fazilleau dies als leserfeindlich einstuft, könnte ebenso angenommen werden, dass Carey den Leser dazu auffordert, diese Strategien zu durchschauen. Auf diese Weise attackiert Carey die Autorität des geschriebenen Worts und fördert einen kritischeren Umgang mit (literarischen) Texten.

\section{Careys Charakterkonzeption: "character [as] a bit of clay"}

Um in seinen Kurzgeschichten die Auswirkungen des bestehenden Wirtschafts- und Gesellschaftssystems kritisch darzustellen, entwirft Carey Charaktere, die sich ähnlich wie Personen im realen Leben von ihrer Umwelt entfremdet haben: "I make very serious 
attempts to produce characters who are not alienated, but then I always feel a bit safer, a lot more confident, when I've made them a little obsessive, a mite neurotic, a smidgin more like me" (73). Denn im Gegensatz zur amerikanischen Mentalität, die Heldenfiguren erlaube, generiere die australische mangels Selbstvertrauen eher Verlierertypen. Selbstironisch fügt Carey hinzu, "it is a relief to be able to blame it [the lack of confidence] on a national characteristic rather than personal failing" (Wachtel 103). Auch wenn Carey mehr Eigenverantwortung begrüßen würde, so erkennt er auch, dass die globalisierte Welt auf die individuelle Entwicklung eher restriktiv als anregend wirkt. Daher begegnet der Leser selten integren, verantwortlichen und selbstbestimmten Charakteren; stattdessen sind Situation und Charakter der höheren Absicht der Kurzgeschichte untergeordnet:

In fiction I do like the notion of a plastic malleable world where a character can be shaped and formed, and everything in the story or the novel is for the needs of the story, so you can shape character like a bit of clay. (Carey in Bennett 195)

In seinen Kurzgeschichten, die Carey selbst als "collection of 'what if' stories" bezeichnet (zitiert in Woodcock 16), ergeht es seinen Charakteren wie Versuchspersonen in einem Experiment, in welchem unter laborähnlichen Bedingungen verschiedene Reaktionsweisen durchgespielt werden. Carey entwirft dabei vier unterschiedliche Versuchsszenarien, die jeweils eine bestimmte Form von Gefangenschaft aufdecken. Diese vier Grundtypen entsprechen Woodcocks Zuordnung von Careys Kurzgeschichten in vier Themenkreise: "American imperialism and culture; capitalism; power and authority; and gender" (18). Trotz diverser Themen und Erzählstrategien lässt sich Gefangenschaft als durchgängiges Motiv aller Kurzgeschichten identifizieren.

\section{Careys Kurzgeschichten: Formen von Gefangenschaft}

Careys Kurzgeschichten sind so konzipiert, dass sie Leser wie Charaktere auf vielfältige Weise gefangen nehmen, um diese zum Ausbruch zu animieren. Während dies seinen Charakteren kaum gelingt, scheint der Leser dazu aufgefordert zu werden, seine eigene Situation $\mathrm{zu}$ überdenken und $\mathrm{zu}$ ändern. Im Folgenden werden die vier Varianten der Gefangenschaft anhand einzelner Kurzgeschichten exemplarisch beschrieben, wobei Strategien zur Lesersteuerung berücksichtigt werden.

\subsection{Amerikanischer Imperialismus: Gefangen im amerikanischen (Alb)traum}

Als erstes Instrument des spätkapitalistischen Systems zur Unterwerfung von Menschen nennt Carey "American imperialism and culture". Diese Form von Gefangenschaft lässt sich besonders deutlich anhand von "American Dreams" darstellen, einer Geschichte, welche die zunehmende Abhängigkeit Australiens von Amerika und die damit verbundene Entwertung der eigenen nationalen Identität aufzeigt. Carey entwirft hier das Bild einer australischen Kleinstadt, deren Bewohner den amerikanischen Traum von Wohlstand und Statussymbolen träumen. In dieser Geschichte vermischen sich verschiedene Erzählstile; sie beginnt wie eine realistische Erzählung in Lawson Tradition, doch beinhaltet sie bizarre und fantastische Elemente: "'American Dreams' takes the orthodox realist story of outback communities and injects a dislocating strangeness into it" (Woodcock 18). Der namenlose Erzähler berichtet, wie sich der kollektive Wunsch nach amerikanischen Verhältnissen zu verwirklichen scheint, als sich im Nachlass eines verstorbenen Bewohners ein perfektes Miniaturmodell der Stadt findet, das zu einer Touristenattraktion wird und amerikanische Dollar in die Stadt strömen lässt. Der Erbauer dieser Replik, Mr Gleason, war so detailverliebt, dass er nicht nur Straßen 
und Häuser nachbaute, sondern auch Miniaturfiguren der Bewohner kreierte. Auf den ersten Blick empfindet der Erzähler das Modell als "the most incredibly beautiful thing I had ever seen in my life" (FMH 153). Hier scheint Carey das romantische Verständnis von Kunst transportieren zu wollen, wonach Kunst Alltägliches als 'schön' vor Augen führt. Doch schon bald zeigt sich die gefährliche Seite von Kunst: Da sich die Dächer der Häuser abnehmen lassen, ermöglicht dieses Kunstwerk ungewollte Einblicke in das Privatleben der Bewohner. Die ursprüngliche Freude am voyeuristischen Blick in das Leben anderer weicht der Angst, die eigenen Abgründe könnten bloßgestellt werden: "If Gleason knew about Mrs. Cavanagh and Craigie Evans (and no one else had), what other things might he know? [...]. We gazed silently at the roofs and felt mistrustful and guilty" (154). Was zunächst affirmativen Charakter zu haben schien, erzeugt paranoide Gefühle. Doch bevor das Modell zerstört werden kann, avanciert es zu einem medialen Ereignis. Die Betroffenen stehen dieser Situation hilflos gegenüber, was sich in der Formulierung zeigt, mit der Carey die Entscheidung des Ministers für Tourismus präsentiert, das Modell für Besucher freizugeben: "The model town and its model occupants were to be preserved" (155). Durch die Passivkonstruktion und die Bezeichnung "occupants" werden die Bewohner zu Gefangenen ihrer eigenen Welt stilisiert.

Zudem begnügen sich die amerikanischen Besucher der Kleinstadt nicht damit, das Modell $\mathrm{zu}$ betrachten, sondern erwarten von den Bewohnern, dieselben Posen einzunehmen, in denen Gleason sie verewigt hat, um mithilfe von Teleskopen Original und Kopie vergleichen zu können. Der Blick durch das Teleskop versinnbildlicht den Prozess des Kolonialisierens durch wertendes Starren. Indem die Amerikaner von einem Hügel aus auf die darunter liegende Stadt blicken, kommt deren Überlegenheit zum Ausdruck. Paradoxerweise ziehen die Amerikaner das Modell der Wirklichkeit vor, und sie zweifeln nicht die Originalität des Modells an, wohl aber die der realen Bewohner - eine verkehrte oder amerikanisierte Welt, in der die amerikanische Vorstellung von Realität wichtiger ist, als die Realität selbst (vgl. 157). Somit wird das Modell zum Korrektiv der 'verfälschten' Realität, die Amerikaner selbst zu Richtern über Echtheit und Plagiat. Der amerikanische Traum entpuppt sich als Albtraum, da "das statische Modell ihre Entwicklungsmöglichkeiten beschneidet" und die "Verwirklichung ihrer materiellen Träume zur Zerstörung ihrer Kleinstadtidylle führt" (Maack 398f). Demnach kann das Modell als Symbol für die Einsicht verstanden werden, dass kulturelle Abhängigkeit zum Verlust individueller und nationaler Identität führt. Am Ende von "American Dreams" impliziert Carey, dass amerikanischer Imperialismus für beide Seiten schädlich ist:

The Americans pay one Dollar for the right to take our photographs. Having spent the money they are worried about being cheated. They spend their time being disappointed and I spend my time feeling guilty that I have somehow let them down by growing older and sadder. (157)

So führt dieses eigentümlich interdependente Verhältnis zwischen 'Imperialmacht' und 'Kolonie' zu einem endlosen Zyklus gegenseitiger (materieller und ideeller) Entwertung.

Careys intensive Auseinandersetzung mit dem Verhältnis zwischen Australien und Amerika soll anhand zwei weiterer Kurzgeschichten verdeutlicht werden. In "Crabs" und "A Windmill in the West" verarbeitet Carey seine Kritik an imperieller und kultureller Dominanz Amerikas zu apokalyptischen Zukunftsvisionen. In beiden Kurzgeschichten befinden sich die Protagonisten in einer aussichtslosen Situation: In "Crabs" werden der 
Protagonist und seine Begleiterin sowie alle anderen Besucher in einem "Star Drive-in Theatre" auf unbegrenzte Zeit festgehalten. Ohne Rücksicht auf seine Begleiterin bereitet er seinen Ausbruch vor, indem er sich in ein Automobil verwandelt. Obwohl seine Flucht zunächst zu gelingen scheint, wird der Protagonist in einer zirkulären Bewegung zurück zum Ausgangspunkt geführt. Wegen seiner egoistischen Haltung nimmt das Opfer Züge eines Täters an. Diese Koexistenz von 'Gut und Böse' in einer einzigen Person wird in einer verwandten Kurzgeschichte motivisch fortgeschrieben. In "A Windmill in the West" muss ein amerikanischer Soldat eine Grenze inmitten einer australischen Wüste bewachen, die er sich als Grenze zwischen Australien und Amerika vorzustellen habe, "although, in fact, it was not [America]" (CS 94). Mit der Zeit wird der Soldat unsicher, welche Seite der Grenze er zu bewachen hat. Seine arbiträre Rolle als Opfer und Täter zugleich zeigt sich in seinem Verhalten gegenüber dem Piloten eines zivilen Flugzeugs, der - wie er selbst - die Orientierung verloren hat und mit leerem Tank notlanden muss. Der Soldat ordert zwar Benzin, doch als der Pilot dankbar winkend in die falsche Richtung fliegt, schießt der Soldat ihn pflichtbewusst ab.

Die vorgestellten Kurzgeschichten zu "American imperialism and culture" haben nicht nur gemeinsam, dass die Protagonisten in eine ausweglose Situationen geraten, sondern zugleich ihre 'Unschuld' verlieren, sobald sie sich selbst in einer scheinbaren Machtposition befinden.

\subsection{Kapitalismus: Gefangen im kapitalistischen System}

Der korrumpierende amerikanische Einfluss ist auch in der zweiten Gruppe von Kurzgeschichten zum Thema "capitalism" spürbar. Die Verknüpfung von Kapitalismus und Macht(missbrauch) stellt eine Variante von Gefangenschaft dar, die wieder Careys Skepsis gegenüber Machtpositionen ausdrückt, ohne zwischen Opfern und Tätern zu polarisieren. Exemplarisch für diesen Typus von Gefangenschaft soll die Titelgeschichte seiner zweiten Sammlung von Kurzgeschichten, War Crimes, vorgestellt werden. Die vorangestellte These, dass Carey seine Leser bewusst manipuliert, um sie zu einer kritischeren Haltung aufzufordern, wird hier bestärkt, da Carey seinen Leser in "War Crimes" zum Mitdenken aufruft - wenn auch auf indirektem Wege. Carey verweist schon im ersten der vierzehn durchnummerierten Erzählabschnitte der Kurzgeschichte auf die Möglichkeit des Lesers, sich ein eigenes Bild zu machen: "In the end I shall be judged" (WC 241). Dieser Satz lässt sich sowohl als Voraussage des Erzählers wie auch des Autors lesen. So fürchtet der Erzähler die Folgen seiner Taten und deren Darstellung durch Dritte, gleichzeitig spricht Carey als Autor den Leser an, der seine Erzählung bewerten wird. Diese Mehrdeutigkeit mag als Verunsicherungsstrategie gewertet werden, doch könnte sie ebenfalls dazu dienen, den Leser zu erhöhter Aufmerksamkeit zu animieren. In Form einer Kritikerschelte erinnert Carey seinen Leser indirekt an dessen Fähigkeit, mit Texten kritisch umgehen zu können:

They will write about me in books and take care to explain me so badly that it is better that I do it myself. They will write with the stupid smugness of middle-class intellectuals, people of moral rectitude who have never seriously placed themselves at risk. [...]. They will write about me as a tyrant, a psychopath, an aberrant accountant, and many other things, but it would never once occur to them that I might know exactly what I am doing. (241)

Anstatt diese Klage wörtlich zu nehmen, kann sie als versteckte Aufforderung an den Leser verstanden werden, die eigene kritische Urteilskraft $\mathrm{zu}$ nutzen. Um zu zeigen, dass der kompetente Leser einiges bewirken kann, verweist Carey ihn auf die Abhängigkeit des 
Autors von seiner Leserschaft. Auch der Erzählers weiß um seinen Wunsch nach Anerkennung: "I cannot begin to tell you how I loathe them, how I have, in weaker moments, envied them, how I longed to be accepted by them..." (241). Während hier die reale Person Carey mit dem fiktiven Erzähler zu einer 'Person' zu verschmelzen scheint, spalten sich die angesprochenen Leser in zwei Gruppen: "they" und "you". An letztere richtet sich der Erzähler/Autor mit verschwörerischer Stimme, als könnten seine Enthüllungen das Verständnis des persönlich angesprochenen Lesers erwirken. Zerrissen zwischen dieser fast erzwungenen Loyalität des Lesers zum Erzähler/Autor und der gleichzeitig wachsenden Abscheu vor den erzählten Taten wird der Leser - der sich dabei ertappt, sich des Vertrauens würdig erweisen zu wollen - zur Passivität gezwungen: "Carey is peremptory here, virtually ordering us to follow the lead he indicates rather than choosing our own method of approach [...], presuming to prolong his own authority over his text and thus usurp ours" (RyanFazilleau 58). Ziel dieser autoritären Leserführung könnte es jedoch sein, den Leser davor zu warnen, allzu leichtgläubig zu sein. Anstatt den Erwartungen zu entsprechen, die von außen an ihn gerichtet werden, sollte er lieber zur unbequemen Gruppe von Lesern zählen, die hier angeblich abgestraft werden.

In den folgenden zwölf Erzählabschnitten berichtet der Ich-Erzähler aus der Retrospektive, dass es sich bei den "war crimes" um Verbrechen in der Geschäftswelt handelt. Gemeinsam mit seinem Geschäftspartner Bart soll der Erzähler eine Firma, die Fertiggerichte herstellt, vor dem Konkurs retten. Das Klischee des 'eiskalten Geschäftsmanns' wird bis auf das Äußerste ausgereizt, indem der Leser am Ende der Kurzgeschichte zum Zeuge von Massenexekutionen von Arbeitslosen wird. Die hilflose Position des Lesers als stillschweigender und handlungsunfähiger Beobachter impliziert eine gewisse Mittäterschaft. Dies ist ein weiterer Hinweis darauf, dass Carey Leser vorzieht, die sich in das Geschehen einmischen, im übertragenen Sinne Zivilcourage zeigen. In einem Interview bekennt er sich zu seinem Wunsch, die Mechanismen des Systems zu entlarven, indem er sie überzeichnet: "[Although the story] pushes things to extremes a bit, [it captures] the logic of business and [...] my view of late capitalism" (Maddocks 39f). Es gilt, sich diesen zu widersetzen.

Als Motto der blutrünstigen Geschichte bietet Carey den bekannten Slogan "BUSINESS MUST GO ON" (WC 281) an. Der Erzähler ist auf merkwürdige Weise gespalten. Einerseits ist er von seiner eigenen Gewalttätigkeit entsetzt, andererseits plädiert er für Verständnis, weil sich diese Taten auf sein instabiles Selbstwertgefühl und traumatische Kindheitserinnerungen zurückführen lassen. Da der Erzähler unter ärmlichen Verhältnissen aufwuchs und Opfer (später Täter) von gewalttätigen Übergriffen wurde, interpretiert er die "war crimes" als Kompensationshandlungen. So bizarr und blutrünstig seine Taten auch sein mögen, erschweren die Einblicke in die Psyche des Täters dessen Verurteilung:

My father lost his hand in a factory. He carried the stump with him as a badge of his oppression by factories. When I was very small I saw that my father had no hand und concluded that my hand would also be cut off when the time came. [...]. My fear was so intense that all communication on the subject was unthinkable. (WC 260)

Hier thematisiert Carey nicht nur die Unterdrückung der (Fabrik-) Arbeiter in der industriellen Arbeitswelt, sondern auch die Auswirkungen auf die psychische Gesundheit der Folge-generation. Tabuisierung führt in diesem Fall dazu, dass die Zustände fortgeschrieben werden. Die Entmachtung des Einzelnen wird noch deutlicher, wenn der Erzähler schildert: "But nothing dulls me to the assault of factories and I carry with me, still, 
the conviction that I will end up at the bottom of the shit pile, powerless against the machines in factories" (264). Anstatt sich für Arbeiter einzusetzen, wird der Erzähler jedoch zum Geschäftsführer der zu sanierenden Fabrik und implementiert ein Regime des Terrors und der Ausbeutung - eine implizite Theorie $\mathrm{zu}$ Ursachen von Gewalt, die an sozialpsychologische Studien erinnert. Dann setzt der Erzähler seine Schilderungen seiner Paranoia und deren Ursachen fort:

I have spent my life in a state of constant fear [...]. I have anticipated disaster at every turn, physical attack at every instant. To be born small and thin and poor, one learns, very quickly, of one's vulnerability. [...]. The extent of my terrible quaking fear was in exact correspondence with the degree of my craziness. For I performed unthinkable acts of cruelty to others. (270)

Als Beispiel für seine Gewalttätigkeiten beschreibt der Erzähler ein Kindheitsereignis, "when I was six years old I threw a cat into an incinerator. It wasn't until the cat came running out the grate at the bottom, burning, screaming, that I had any comprehension of what I had done" (273). Aus einem Akt der Neugierde erwuchs eine Form von Grausamkeit, die mit dem Bild eines Sechsjährigen kaum zu verbinden ist. Seine reale Tat verewigt sich in wiederkehrenden Albträumen, "the burning cat still runs through my dreams searing me with its dreadful knowledge" (273). Als Bart 'versehentlich' einen Jungen erschießt, interpretiert der Erzähler diese Situation als "Bart's burning cat" (273), aber auch als unvermeidbaren Nebeneffekt der Geschäftswelt: "It is the nature of business that as a result of your decisions some people will starve and others be killed (273f). Die Gewalt, die als unabwendbar dargestellt wird, eskaliert in den letzten drei Abschnitten. Im zwölften Abschnitt wird der erschossene Junge als diffuse Warnung an die Arbeitslosen aufgehängt: "Let that warn the bastards. [...]. The unemployed shall buzz with powerless rage" (276). Barts Entsetzen über den unabsichtlich zu Tode gekommenen Jungen ist nicht mehr zu spüren, als die Leiche zu einem Emblem der Ohnmacht aller Arbeitslosen umfunktionalisiert wird. Anschließend findet eine öffentliche Exekution des Leiters der Buchhaltung statt, da der Erzähler annimmt, dass Sergei Gelder veruntreut hat. Ohne Rücksprache mit seinem Partner Bart bereitet er lustvoll die Tötung vor: "It will be a most inventive execution, far more interesting than his dull childish cheating" (276). Später stellt sich heraus, dass Sergei auf Barts Anweisung hin Gelder abgezogen hat, um die Fabriken so umzubauen, dass sie auf den Erzähler weniger bedrohlich wirken:

They have worried about aeshetics. Areas of peaceful blue and whole fields of the most lyrical green. In these ideal conditions people perform insulting functions, successfully imitating the functions of mid-twentieth century machinery. This is Bart's and Sergei's masterpiece, their gift to me. (278)

So wie Carey in "American Dreams" das Miniaturmodell der Kleinstadt zu einem Kunstwerk erklärt, welches die Bewohner zu Marionetten der Touristenwünsche werden lässt, so werden in "War Crimes" die umgebauten Fabriken zu seinem künstlerischen Meisterwerk, in welchem die Arbeiter in festgeschriebenen Abläufen gefangen sind. Denn diese kosmetischen Veränderungen vermögen es nicht zu vertuschen, dass die Arbeiter weiterhin monotone Tätigkeiten zu verrichten haben. Somit wird nicht nur der Umbau, sondern auch der Tod von Sergei sinnlos. Der Verlust seines Freundes Sergei löst in Bart Tötungswünsche aus, und aus Angst, sein Geschäftspartner könnte diesen Wünschen nachgeben, ordnet der Erzähler die Exekution der Arbeitslosen an, "it will give him a use for his rage" (280f). Den 
Impuls des Lesers, sich völlig vom Erzähler zu distanzieren, vereitelt Carey, indem er diesen erklären lässt, dass das Böse in allen Menschen steckt: "And I am not mad, but rather opened the door you all keep locked with frightened bolts and little prayers. I am more like you than you know" (280). Der letzte kurze Abschnitt der Geschichte fungiert als grausames Finale. Die Arbeitslosen werden aus dem Gelände getrieben und von Arbeitern mit Flammenwerfern bei lebendigem Leibe verbrannt. Der Erzähler beobachtet die Szene mit einem Fernglas und stellt so Distanz zum Geschehen her. Hier vollendet sich sein diabolisches 'Meisterwerk', und er sieht sich inmitten einer endlosen Abfolge von Tyrannen: "As I watched men run through the heat burning other men alive, I knew thousands of men had stood on hills or roofs and watched scenes of terrible destruction, the result of nothing more than their fear and their intelligence" (281f). Gefühlskalt analysiert er als Ursache der Verbrechen eigene Ängste. Seine Handlungen sind demnach nicht unabwendbar, sondern hätten durch die Konfrontation mit den eigenen Ängsten vermieden werden können. Das apokalyptische Szenario garniert Carey mit den Schlussworten: "I wished I had been born a great painter. [...]. I would have stood aloft, a judge, rather than wearily kept vigil on this hill, hunchbacked, crippled, one more guilty fool with blood on his hands" (282). Nochmals präsentiert sich der Erzähler als Opfer, das aufgrund seiner Herkunft und traumatischen Kindheit zu einem Verbrecher wurde. Doch mit "War Crimes" zeigt Carey eindrucksvoll, wozu es führen kann, wenn man sich seiner Verantwortung entzieht und seinen destruktiven Impulsen nachgibt. Jeder Mensch, so scheint die These, erhält die Möglichkeit, sich zu entscheiden, ob er das repressive System unterstützen oder sich ihm entgegen stellen will.

\subsection{Macht und Autorität: Gefangen in zugewiesenen Rollen}

Als dritte Variante von Gefangenschaft nennt Carey "power and authoritiy", die in der Kurzgeschichte "Life \& Death in the South Side Pavilion" besonders plakativ dargestellt wird. Die Kurzgeschichte erinnert an "War Crimes", da sie ebenso aus der Sicht eines namenlosen männlichen Erzählers geschildert wird, die Form einer Beichte annimmt und in Erzählabschnitten durchnummeriert ist. Am Anfang der Kurzgeschichte erklärt der Erzähler die Absicht, seinen Job zu kündigen, der darin besteht, eine Herde von Pferden davor zu bewahren, in einen Pool zu fallen und zu ertrinken. Sein eigenes Befremden über diese Tätigkeit wird im vierten Abschnitt deutlich, der aus nur einem Satz in Großbuchstaben besteht: "I AM HERE TO STOP THE HORSES FALLING INTO THE SWIMMING POOL" (52). Weitere Umstände bleiben mysteriös. Während die Namenlosigkeit des Arbeitgebers den Eindruck einer anonymen, gesichtslosen Macht noch verstärkt, steht die des Erzählers für seine Ohnmacht. Als Absender seines Gesuchs kann er nur eine Bezeichnung angeben, die seinen geringen Status schon im Titel benennt: "Shepherd 3rd Class in the South Side Pavilion" (CS 51). Aus der Sicht des dubiosen Arbeitgebers setzt sich seine Identität ausschließlich aus Tätigkeit, Status- und Ortsangabe zusammen. Dahingegen trägt seine Freundin den Namen Marie, und dieser Unterschied ist zugleich mit anderen Privilegien verbunden: Sie kann den Pavillon jederzeit verlassen und definiert die Bedingungen des Zusammenseins. Schließlich sieht er sich von ihr vor die Wahl gestellt: "Either I leave the horses or she will leave me" (52). Marie tritt dem Leser als willkürlich und rücksichtslos entgegen. Hatte sie dem Erzähler zunächst den Job vermittelt, erwartet sie nun, dass er ihr folgt - ohne dass für ihn oder den Leser deutlich würde, wohin sie ihn führen wird. 
Der Erzähler sieht sich gefangen in einer absurden Lage. Denn obwohl er unter jedem einzelnen Todesfall leidet, kann er den Ort des Geschehens verlassen, wenn das letzte Tier umgekommen ist. Der Zustand des Erzählers verschlimmert sich, als er erkennt, dass jeder Liebesakt mit Marie und die damit verbundene Unachtsamkeit zu einem weiteren Todesfall führt: "EVERY TIME I FUCK MARIE I KILL A HORSE" (54). Seine Schuldgefühle lösen Potenzproblemen aus: "And now that I am unable to make love she thinks it is because I have an unnatural attraction to the horses and that I find her unattractive in comparison. But I am unable to make love because every time I make love a horse falls into the pool" (54). Anstatt ihren Liebhaber zu trösten, unterstellt Marie ihm den latenten Wunsch nach Sodomie. In Verbindung mit der Thematik von Macht und Ohnmacht äußert sich Carey hier deutlich zum Geschlechterverhältnis. Interessanterweise werden dem Erzähler zunächst typisch 'weibliche' Eigenschaften wie Fürsorge, Mitleid und Selbstaufgabe zugeschrieben, wohingegen Marie Handlungsweisen zeigt, die traditionellerweise als typisch 'männlich' gelten; so ist sie in ihren Entscheidungen autonom und handelt ichbezogen. Auch zeigt der Erzähler ein stärkeres Bedürfnis, sich mitzuteilen, worauf Marie mit kränkendem Spott reagiert: Als der Erzähler beispielsweise vermutet, die Geräusche des Geschlechtsakts könnten bei den Pferden Panik auslösen und diese in den Pool treiben, antwortet Marie: "You attribute great power to your cock" (54). Mit dieser Aussage unterstreicht sie die Ohnmacht des Erzählers, der an dieser Stelle schon unter Impotenz leidet. Als sie ihn schließlich verlässt, verbessert sich der physische und emotionale Zustand des Erzähler: "Her absence has cured my limp cock more quickly and effectively than either of us could have guessed" (54). Mit seiner Manneskraft verstärkt sich sein Drang, seine Gefangenschaft zu verlassen, und seine Fürsorge weicht Indifferenz: "At this moment I am prepared to fuck until the pool is full of horses" (55). Während sich hier der Eindruck verstärkt, dass gelebte Sexualität zerstörerisch wirkt, spielt Carey gleichzeitig mit dem Klischee, dass Männlichkeit mit Egoismus und Autonomie verbunden ist, d.h. seine vorherige Umkehrung von 'männlichen' und 'weiblichen' Eigenschaften wird hier revidiert. Mutwillig treibt der Erzähler die restlichen Pferde in den Pool: "It was sickingly easy. They fell onto the water like overripe fruit from a tree..." (55). Diese Szene erinnert an die Massenexekutionen am Ende von "War Crimes," auch wenn der Tod der Tiere weniger drastisch erscheint und die Situation einen ausgeprägt surrealen Charakter hat. Eine weitere Parallele drängt sich auf, wenn sich die Erinnerungen an die toten Pferdeleiber im überfüllten Pool ebenso in die Träume des Erzählers schleichen, wie das Bild der misshandelten Katze in die des mordenden Kapitalisten. Doch "Life \& Death in the South Side Pavilion" endet nicht mit den Albträumen, die den Schlafenden heimsuchen, sondern mit dem albtraumhaften Charakter des realen Lebens: Die Grausamkeit des Erzählers führt nicht zur Erlösung, sondern nur wieder an den Anfang seiner Zwanglage zurück: "They have brought replacements" (55). Die verzweifelte Bitte des Erzählers, von dieser widersinnigen Aufgabe entbunden zu werden, wird ignoriert. Er bleibt zurück, gefangen in einer aufdoktrinierten, lebenslangen Tätigkeit.

Die letzte Gruppe von Kurzgeschichten, die sich explizit mit Genderfragen befasst, nimmt die Verknüpfung von Sexualität und Zerstörung wieder auf.

\subsection{Gender: Gefangen in sich auflösenden Geschlechterrollen}

Als vierte Variante von Gefangenschaft führt Carey "gender" an. In "Peeling," einer seiner frühesten Erzählungen, verwischen sich die Grenzen zwischen den Geschlechtern. Die 
Geschichte handelt von einem männlichen älteren Erzähler, der seine Beziehung zu einer jungen Frau, Nile, beschreibt. Wieder steuert Carey das Leserverhalten, indem der Erzähler warnt, "our relationship is beyond analysis" (FMH 34). Wie schon in früheren Kurzgeschichten lässt sich diese Warnung als Aufforderung an den Leser deuten, sich aktiv mit der präsentierten Beziehung auseinander zu setzen. Dass "Peeling" nicht aus der Retrospektive erzählt wird, sondern der Leser Zeuge der Beziehung wird, an deren Ende sich Nile im wörtlichen Sinne auflöst, erhöht die Betroffenheit des Lesers und mag darauf abzielen, ihn dazu anzuregen, über die eigene Geschlechterrolle und partnerschaftliche Beziehungen zu reflektieren.

Einleitend erfährt der Leser von Niles eigentümlichen Angewohnheit, Puppen zu sammeln, um sie in zeremonieller Weise zu verstümmeln: "Those which still have hair she plucks bald, and those with eyes lose them, and those with teeth have them removed and she paints them, slowly, white" (35). Diese ritualisierte Form der Gewalt führt zu einer Art Entfeminisierung, und die weiße Farbe steht nicht für Unschuld, sondern Bedeutungslosigkeit: "White [...] has no appeal to her, it is simply that it says nothing" (35). Niles destruktives Verhalten, das ihre gestörte Weiblichkeit symbolisiert, führt in die Genderproblematik ein. Die Verwirrung um das eigene Geschlecht und um Geschlechtszugehörigkeit verstärkt sich, als der Erzähler bekennt, die Farbe weiß nicht zu mögen, sondern blau vorzuziehen: "I would prefer a nice blue [...]. Something a little more feminine" (35). Da üblicherweise die Farbe blau für Jungen und rosa für Mädchen ausgewählt wird, wirkt diese Aussage irritierend. Erst nach diesen Vorüberlegungen beschreibt der Erzähler seine Beziehung zu Nile, die ihn im Haushalt unterstützt und mit ihm Mahlzeiten zu sich nimmt. Bald gesteht der Erzähler ein, dass er zu einem späteren Zeitpunkt Sex mit Nile haben möchte. Die folgende Metapher des schichtweise Entblößens steht nicht für den Prozess des Kennenlernens, sondern eher für den Wunsch des Erzählers, sich seiner Partnerin zu bemächtigen: "Then it will be time to move on to other more intimate things, moving layer after layer, until I discover her true colours, her flavours, her smells. The prospect of so slow an exploration excites me and I am in no hurry, no hurry at all" (35f). So wird Nile zu einem Objekt seiner sexuellen Phantasien, in denen Zeitpunkt und Art des Geschlechtsakts seinen Wünschen obliegen. Die Übergriffe des Erzählers auf Nile werden von Übergriffen auf den Leser in Form von suggestiven Leseranreden ergänzt. Die Situation entgleist, als Nile ihm offenbart, dass sie an illegalen Abtreibungen beteiligt ist. Der Erzähler ist nicht wegen ihrer Tätigkeit entrüstet, sondern weil sie sich nicht an die angemessene Reihenfolge der Gesprächsinhalte hält: "It is bad. I had wished to take things slowly. [...] But it is all coming too fast, all becoming too much" (39). Kontrollsucht erscheint hier als zentrales Prinzip, da der Erzähler vom richtigen Ablauf, Zeitpunkt und seiner Verzögerungstechnik besessen ist. Gleichzeitig will er sich Niles bemächtigen. Dieser Widerspruch zwischen künstlicher Distanz und gewaltsamer Intimität prägt das hier präsentierte Männerbild. Anstatt das Tempo zu drosseln, verliert er die Kontrolle über sich selbst: "I am doing what I had planned not to do: rush" (40). Während Nile weitere Geheimnisse preisgibt, entkleidet der Erzähler sie mit zunehmender Erregung. Schließlich sitzt sie nackt vor ihm, übrig ist nur ein Ohrring. Obwohl Nile sich wehrt, zieht er heftig am Schmuckstück, das die Funktion eines Reißverschlusses zu haben scheint: "It is not, it would appear, an earring at all, but a zip or catch of some sort. As I pull, her face, then her breasts, peel away" (42). Zum Vorschein kommt ein junger Mann mit ihrem Gesicht und Haar. Überrascht über ihre eigene Metamorphose greift Nile nach ihrem Geschlechtsteil, "she takes her penis in her hand, curious, kneading it, watching it grow. I watch fascinated" 
(42). Hier werden tabuisierte Themen wie Masturbation und Voyeurismus angesprochen. Männliche Sexualität hat einen narzistischen oder homoerotischen Aspekt, "a displaced desire for a hidden male body, an awareness that rather than being biologically determined, gender identity is a culturally imposed demarcation which splits men off from their polymorphus potentialities" (Woodcock 31). Anstatt seine homo- oder bisexuellen Neigungen ausleben zu können, wird der Mann auf eine eindeutige Sexualität zurück geworfen.

Als der Erzähler einen weiteren Ohrring entdeckt, reißt er auch an diesem, und es erscheint eine mädchenhafte Ausgabe von Nile als Frau, die Strümpfe und einen Strumpfbandhalter trägt. Diese Frau/Mann Phantasie enthält auch die Vorstellung der Frau als Objekt, deren Verfügbarkeit durch klassischen Fetische markiert wird. Als der Erzähler die Strümpfe abrollt, verschwinden ihre Beine, er greift nach ihren Haaren, die nur eine Perücke sind und einen kahlen Kopf entblößen. Schließlich lässt er sie fallen, und Niles Körper zerfällt: "There is a sharp noise, rather like a breaking glass. Bending down I discover among the fragments a small doll, hairless, eyeless, and white from head to toe" (43). Am Ende der Geschichte sieht der Erzähler sich mit einer weißen Puppe konfrontiert, als würden seine eigenen Phantasien in die Obsessionen seines Sexualobjekts übergehen.

Die Geschichte ist mehrdeutig: Sie kann als "a deconstruction of male mythologies about women" interpretiert werden (Woodcock 30). Vor allem Niles Identität bleibt unklar: Nile muss keine reale Frau sein, sondern es könnte sich um eine Wunschphantasie eines älteren, einsamen Mannes handeln. Ebenso könnte sie eine Prostituierte sein, die zunächst auf Kundenwünsche eingeht, sich aber dann dagegen auflehnt. Weitere Fragen bleiben ungeklärt: Ist Niles besessene Verstümmelung von Puppen ein Symptom ihrer Panik, von einem Mann sexuell missbraucht und somit gesichts- und bedeutungslos zu werden? Kann der Sexualakt unter bestimmten Umständen eine ähnlich zerstörerische Wirkung wie eine Abtreibung haben? Oder steht die Puppe für die sexuelle Enttäuschung des Erzählers, der trotz pornographischer Phantasien zuletzt allein, bindungslos und unbefriedigt bleibt?

Der Vorgang des Entkleidens kann auch metafiktional als Freilegen verschiedener Deutungen der Erzählung interpretiert werden. Die anfängliche Warnung, die dargestellte Beziehung könne nicht analysiert werden, könnte ein Aufruf sein, es dennoch zu versuchen. Dann stünde die Beziehung zwischen dem Erzähler und Nile nicht nur programmatisch für das Verhältnis zwischen Mann und Frau, das es aufgrund sich auflösender Geschlechterrollen neu zu definieren gilt, sondern auch für das zwischen Autor und Leser, das neue Lesestrategien fordert.

Die zuvor demonstrierte Lesart von Peter Careys Kurzgeschichten als Lehrstücke entkräftet den eingangs genannten Vorwurf, Carey wolle seinen Leser absichtlich in die Irre führen und eröffnet einen neuen Blick auf seine Erzählungen: Careys Kurzgeschichten, in denen verschiedene Varianten von Gefangenschaft und deren Ursachen präsentiert werden, sind nicht nur brillant konstruierte Erzählungen, die den Leser zunächst in ein erzählerisches Labyrinth führen, sondern fordern diesen gleichzeitig zu einer kritischeren (Lese-) Haltung heraus. Wer sich gegängelt fühlt, mag sich zur Wehr setzen - vermutlich liegt selbst diese Reaktion in Careys Absicht. 


\section{Bibliographie}

Bennett, Bruce. Australian Short Fiction: A History. St. Lucia: Queensland University Press, 2002.

Carey, Peter. Collected Stories. London: Faber and Faber, 1995. [CS]

--- , The Fat Man in History. St. Lucia: University of Queensland Press, 1974. [FMH]

--- , War Crimes. St Lucia: University of Queensland Press, 1979. [WC]

Fletcher, M.D. "The Theme of Entrapment in Peter Carey's Fiction". Australian Literature Today: Indian Society for Commonwealth Literature. R.K. Dhawan. D. Kerr (Hrsg.). Neu Delhi, 1993.

Hassall, A.J. "Telling Lies and Stories: Peter Carey's Bliss". Modern Fiction Studies. 35.4 (1989): 637-653).

Ikin, Van. "Answers to Seventeen Questions: An Interview with Peter Carey". Science Fiction: A Review of Speculative Literature. 1.1 (1977).

Kane, Paul. "Postcolonial/Postmodern: Australian Literature and Peter Carey". World Literature Today: A Quarterly of the University of Oaklahoma. 67.3 (1993): 512-22.

Maack, Annegret. "Die Auseinandersetzung mit amerikanischen Einflüssen in der australischen short story". Anglistentag 1983 Konstanz: Vorträge. Gießen: Hoffmann, $1984:$ 391-404.

Maddocks, John. "Bizarre realities: An Interview with Peter Carey". Southerly. 41 .1 (1981).

Neilsen, Philip. "Waiting for the Barbarians: An Interview with Peter Carey". Linq. 15.3 (1987): 6673.

Ryan-Fazilleau, Suzan. "One-Upmanship in Peter Carey's Short Stories". Journal of the Short Story in English. 16 (1991): 51-63.

Todd, Richard. "Narrative trickery and Performative Historiography: Fictional Representation of National Identity in Graham Swift, Peter Carey, and Mordecai Richler". Magical Realism: Theory, History, Community. Lois Zanora. W. Faris (Hrsg.). Durham, 1995.

Wachtel, Eleanor. "'We really make ourselves up': An Interview with Peter Carey". Australian and New Zealand Studies in Canada. 9 (1993): 103-105.

Wilde, William. Hooton, Joy. Andrews, Barry (Hrsg.). The Oxford Companion to Australian Literature. Melbourne: OUP Australia, 1985.

Wilding, Michael (Hrsg.). "Introduction". The Oxford Book of Australian Short Stories. Melbourne: Oxford University Press Australia, 1994: ix-xvii.

Woodcock, Bruce. Peter Carey. Contemporary World Writers. John Thieme (Hrsg.). Manchester: Manchester University Press, 1996 